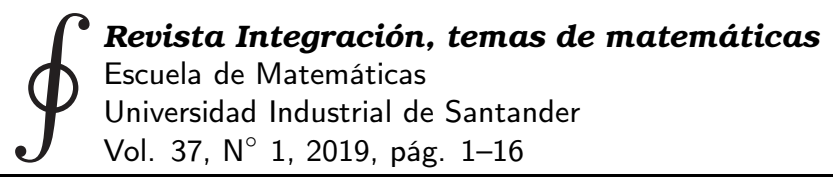

DOI: http://dx.doi.org/10.18273/revint.v37n1-2019001

\title{
An elliptic equation with random potential and supercritical nonlinearity
}

\author{
L. Cioletti $^{a}$, L. C. F. Ferreira ${ }^{b *}$, M. Furtado $^{a}$ \\ ${ }^{a}$ Universidade de Brasília, Departamento de Matemática, Brasília, Brazil. \\ ${ }^{b}$ Universidade Estadual de Campinas, IMECC - Departamento de Matemática, \\ Campinas, Brazil.
}

\begin{abstract}
We are concerned with a nonhomogeneous elliptic equation with random potential and supercritical nonlinearity. Existence of solution is obtained almost surely for a class of potentials that includes continuum and discrete ones. Also, we provide a law of larger numbers for the obtained solutions by independent ensembles and estimate the expected value for their $L^{\infty}$-norms.

Keywords: Elliptic equations, Random potentials, Random nonlinear equations.

MSC2010: 47B80, 60H25, 35J60, 35R60, 82B44, 47H10.

\section{Una ecuación elíptica con potencial aleatorio y no linealidad supercrítica}

Resumen. Estamos interesados en una ecuación elíptica no homogénea con potencial aleatorio y no linealidad supercrítica. Obtenemos la existencia de solución casi seguramente para una clase de potenciales que incluye continuos y discretos. Además, proporcionamos una ley de grandes números para las soluciones obtenidas por conjuntos independientes y estimamos el valor esperado para sus normas $L^{\infty}$.

Palabras clave: Ecuaciones elípticas, potenciales aleatorios, ecuaciones no lineales aleatorias.

\footnotetext{
*Email: lcff@ime.unicamp.br

Received: 11 March 2018, Accepted: 3 May 2018.

To cite this article: L. Cioletti, L. C. F. Ferreira and M. Furtado, An elliptic equation with random potential and supercritical nonlinearity, Rev. Integr. temas mat. 37 (2019), No. 1, 1-16. doi: 10.18273/revint.v37n1-2019001.
} 


\section{Introduction}

A class of models that appears naturally in a wide number of phenomena are the random differential equations. This occurs because randomness is a powerful tool and concept to control complex systems involving a large number of variables and particles. The basic idea is to describe complex systems by means of their statistical properties. Another kind of phenomena are those governed by quantum mechanics and the uncertainty principle. In this direction, we have Schrödinger equations, and their random versions, which are the core in the study of condensed matter.

The semilinear Schrödinger equation reads as

$$
i h \frac{\partial \psi}{\partial t}=-h^{2} \Delta \psi+V(x) \psi-|\psi|^{p-1} \psi, \quad x \in \mathbb{R}^{n},
$$

where $t \in \mathbb{R}, n \geq 3,1<p<\infty, h$ is the Planck constant and $i$ is the imaginary unit. When looking for standing wave solutions, namely those with the special form $\psi(x, t):=e^{-i \frac{E}{h} t} u(x), E \in \mathbb{R}$, we are leading to solve an equation of the type

$$
-\Delta u+V(x) u=|u|^{p-1} u, \quad x \in \mathbb{R}^{N} .
$$

From the physical viewpoint, the function $V$ is the potential energy, and therefore the force acting on the system is given by $F(x)=-\nabla V(x)$. In [20] the author considered a singularly perturbed version and obtained the existence of solution by assuming that $V$ is such that

$$
0<\inf _{x \in \mathbb{R}^{n}} V(x)<\liminf _{|x| \rightarrow+\infty} V(x) .
$$

In [8], the authors showed that the same holds if $V$ has a local minimum. Later, many authors considered multiplicity and qualitative properties of solutions (see [1], [2] and references therein).

The main interest of this paper is to study situations where the potential $V$ is not deterministic. We show existence and probabilistic properties for a nonhomogeneous random version of (2), namely

$$
\left\{\begin{aligned}
-\Delta u+V_{\omega}(x) u & =b(x) u|u|^{p-1}+g(x), & & \text { if } x \in U ; \\
u & =0, & & \text { if } x \in \partial U,
\end{aligned}\right.
$$

where $1<p<\infty, V_{\omega}$ is a random variable, $U \subset \mathbb{R}^{n}$ is a bounded domain and the terms $b, g \in L^{\infty}(U)$ are deterministic. In the case $V \equiv 0$ Pohozaev-type identities provide nonexistence of positive solutions for (2) with critical and supercritical variational values $\frac{n+2}{n-2} \leq p<\infty$. So, it is natural to consider a nonhomogeneous term on the righthand side of (3). Here we desire to cover not only high-powers for $p$, but also the effect on the random term $V_{\omega}$. Our results work well for $b \equiv 1$, and in this case (3) is precisely the perturbation of (2) by the non-homogeneous term $g$. Also, the boundedness of $U, b, g$ are not essential and could be circumvented by working in other settings, such as homogeneous weighted $L^{\infty}$-spaces, $P M^{a}$-spaces and anisotropic Lebesgue spaces (see, e.g., [11], [12], [13], [14], [15]). However, here this condition will simplify matters a bit. The random potential $V_{\omega}$ is constructed as follows: given a continuous function $f: \mathbb{R}^{N} \rightarrow \mathbb{R}$, we consider

$$
V_{\omega}(x):=\int_{U} f(x-y) d \mu_{\omega}(y)
$$

[Revista Integración, temas de matemáticas 
where $\mu_{\omega}$ is a $\mathcal{M}(U)$-valued random variable and $\mathcal{M}(U)$ denotes the set of all Radon measures on $U$ with finite variation.

We present here some examples of (4) that have been treated in the literature (see e.g. the review [18]). We first consider a model of an unordered alloy, that is, a mixture of several materials with atoms located at lattice positions. Assuming that the type of atom at the lattice $i \in \mathbb{Z}^{n}$ is random, we are led to consider potentials of the type

$$
V_{\omega}(x)=\sum_{i \in \mathbb{Z}^{n}} q_{i}(\omega) f(x-i),
$$

where the random variables $q_{i}$ describe the charge of the atom at the position $i$ of the lattice. Other examples can be obtained by considering materials like glass or rubber, where the position of the atoms of the material are located at random points $\eta_{i}$ in space. By normalizing the charge of the atoms, the suggested potential is formally

$$
V_{\omega}(x)=\sum_{i \in \mathbb{Z}^{n}} f\left(x-\eta_{i}(\omega)\right),
$$

where the $\eta_{i}(\omega)$ are random variables which localize the atoms in space.

The class of potentials allowed here is sufficiently large to consider many known models. For example, the case of glass considered in (6) can be obtained by taking the random point measure $\mu_{\omega}=\sum_{i} \delta_{\eta_{i}(\omega)}$. Actually, for this choice of the measure we have that

$$
\sum_{i \in \mathbb{Z}^{n} \cap U} f\left(x-\eta_{i}(\omega)\right)=\int_{U} f(x-\eta) d \mu_{\omega}(\eta)
$$

Also, a combination of potentials like (5) and (6), namely

$$
V_{\omega}(x)=\sum_{i \in \mathbb{Z}^{n} \cap U} q_{i}(\omega) f\left(x-\eta_{i}(\omega)\right)
$$

is also covered by (4) with $\mu_{\omega}=\sum_{i \in \mathbb{Z}^{n} \cap U} q_{i}(\omega) \delta_{\eta_{i}(\omega)}$. It is not difficult to see that we can also consider other models like, e.g., the Poisson model (see [18] for more examples).

The models (5) and (6) correspond to discrete measures $\mu_{\omega}$ for which results about localization, spectral properties and decays can be found in [18], [21]. For Schrödinger equations defined in a lattice, i.e. $x \in \mathbb{Z}^{n}$, we refer the reader to [5]. Considering a random time-dependent potential for (1), the authors of [3] studied asymptotic behavior of solutions by showing convergence for Gaussian limits when the two-point correlation function of the potential is rapidly decaying. Still for time-dependent random potentials, scaling limits for parabolic waves in random media were investigated in [10]. Another type of random equations are the parabolic ones, for which we refer to the works [4], [6], [7] and their references. In fact, the authors of [4] extended regularity properties (Kalita's results) to the stochastic case by considering quasilinear parabolic systems under a random perturbation of Itô type (see [16] for further results on stochastic PDEs).

In this paper we show that a solution for the nonlinear elliptic PDE (3) exists almost surely (or not) depending on the $\nu$-measure of the interval $\left[0, k_{0}\|f\|_{\infty}^{-1}\right)$, where $\nu$ is the probability measure induced on $\mathbb{R}$ by the random variable $\omega \mapsto\left|\mu_{\omega}\right|$ and $k_{0}$ is a given

Vol. 37, $\left.\mathrm{N}^{\circ} 1,2019\right]$ 
constant (see Theorem 3.2). For that, we obtain Proposition 3.1 which seems not to be available in the literature even for the deterministic version of (3). Solutions are understood in an integral sense based on Green functions. In Remark 3.3 and Corollary 3.4 , we give some examples of continuum random potentials covered by our results. Since we are considering $L^{\infty}$-valued random solutions, it is natural to ask about the expected value of the $L^{\infty}$-norm of solutions. In Theorem 3.5, we provide an estimate for this value depending on the size of the potential. Moreover, we obtain a law of larger numbers for solutions obtained by independent ensembles. It is worth to mention that, when dealing with the random variable $\omega \mapsto u(x, \omega)$ that maps an element of $\Omega$ in the solution of (3) associated with the random potential $V_{\omega}$, we need to consider some known concepts of real random variables in a more general setting (see Section 2 for more details).

As a further comment, we observe that the random potentials considered here are built from a very general probability space. In this setting it does not always make sense to ask what is the probability that the problem (3) has a solution in $L^{\infty}(U)$. In order to give some sense to this question we should restrict ourself to probability spaces $(\Omega, \mathcal{F}, \mathbb{P})$ and random potentials $V$ where the set

$$
\left\{\omega \in \Omega \text { : the problem (3) has a solution in } L^{\infty}(U)\right\}
$$

is an event (measurable). Working in such probability spaces, Theorem 3.2 gives us immediately a lower bound for the probability of the non-linear problem (3) having a solution.

The manuscript is organized as follows. In the next section, we introduce some notations, basic definitions and give some properties for an integral operator associated with the random potential $V_{\omega}$. The main results are stated and proved in Section 3.

\section{Preliminaries and notation}

Throughout this paper $(\Omega, \mathcal{F}, \mathbb{P})$ denotes a given complete probability space. If $(E, \mathcal{E})$ is a measurable space, any $(\mathcal{F}, \mathcal{E})$-measurable function $X: \Omega \rightarrow E$ will be called a $E$-valued random variable. We use the abbreviation a.s. for almost surely or almost sure.

Let $U \subset \mathbb{R}^{n}$ be a bounded domain. We adopt the standard notation $\mathcal{M}(U)$ to denote the set of all Random measures on $U$ with finite variation and we call $\mathscr{B}(\mathcal{M}(U))$ the $\sigma$ algebra of the borelians of $\mathcal{M}(U)$ generated by the total variation norm. The space of all bounded continuous real-valued functions defined on $U$ will be denoted by $B C(U)$. Since $B C(U)$ is a metric space with the supremum norm, when we refer to a $B C(U)$-valued random variable, the considered $\sigma$-algebra is always the one generated by the borelians. Similarly to a $\mathcal{X}$-valued Borel random variable $X: \Omega \rightarrow \mathcal{X}$, where $\mathcal{X}$ is an arbitrary metric space.

The random potentials considered here are the $B C(U)$-valued random variables defined as follows. Take any random variable $X: \Omega \rightarrow \mathcal{M}(U)$ (which is simply a random measure in $\mathcal{M}(U))$ and a fixed function $f \in B C\left(\mathbb{R}^{n}\right)$. Then, for $\mu_{\omega}=X(\omega)$, the function $V: \Omega \rightarrow B C(U)$ defined by

$$
V_{\omega}(x):=\int_{U} f(x-y) d \mu_{\omega}(y), \quad x \in U,
$$

[Revista Integración, temas de matemáticas 
is a $B C(U)$-valued random variable that will be called a random potential. To see that $V$ is a well-defined $B C(U)$-valued random variable, is enough to consider the mapping $T_{f}: \mathcal{M}(U) \rightarrow B C(U)$ given by

$$
T_{f}(\mu)(x)=\int_{U} f(x-y) d \mu(y), \quad x \in U,
$$

and to observe that $V=T_{f} \circ X$. In fact, if we denote by $|\mu|$ the total variation of the measure $\mu$, we have the inequality

$$
\left\|T_{f}(\mu)\right\|_{\infty}:=\sup _{x \in U}\left|T_{f}(\mu)(x)\right| \leq\left(\sup _{x \in \mathbb{R}^{n}}|f(x)|\right) \mathbf{|} \mu \mid \mathbf{|}
$$

which implies that $T_{f}(\mu)$ belongs to $L^{\infty}(U)$. Also, proceeding as in (8) and using dominated convergence theorem, one can show that $T_{f}(\mu)$ is a continuous function, and so Borel measurable. It follows that $V$ is a composition of two Borel measurable functions and a $B C(U)$-valued random variable.

Let $(U, \mathscr{B}, \mu)$ be a measure space. For a measurable function $f$ we define

$$
\|f\|_{L^{\infty}(U, d \mu)}=\inf \{a \geq 0: \mu(\{x:|f(x)|>a\})=0\},
$$

and the space $L^{\infty}(U, \mathscr{B}(U), \mu)$ as the set

$$
\left\{f: U \rightarrow \mathbb{R}: f \text { is Borel measurable and }\|f\|_{L^{\infty}(U, d \mu)}<\infty\right\} .
$$

When $d \mu=d x$ is the Lebesgue measure in $U \subset \mathbb{R}^{n}$, we simply denote $L^{\infty}(U)=$ $L^{\infty}(U, \mathscr{B}(U), d x)$. Although we are assuming that $f \in B C\left(\mathbb{R}^{n}\right)$, most of the results presented here are also valid if we suppose only the weaker condition $f \in$ $\bigcap_{\mu \in \mathcal{M}(U)} L^{\infty}(U, \mathscr{B}(U), \mu)$.

In order to state some convergence results, we need to use the notion of Bochner integrals. Let $\left(\mathcal{X},\|\cdot\|_{\mathcal{X}}\right)$ be a Banach space and $(\Omega, \mathcal{F}, \mathbb{P})$ be a probability space. If $X: \Omega \rightarrow \mathcal{X}$ is a $\mathcal{X}$-valued Borel random variable such that $X=Y$ a.s. in $\Omega$, where $Y: \Omega \rightarrow \mathcal{X}$ is a $\mathcal{X}$-valued Borel random variable with $Y(\Omega) \subset \mathcal{X}$ separable, and

$$
\int_{\Omega}\|X(\omega)\|_{\mathcal{X}} d \mathbb{P}(\omega)<\infty
$$

then there exists a unique element $\mathbb{E}[X] \in \mathcal{X}$ with the property

$$
\ell(\mathbb{E}[X])=\int_{\Omega} \ell(X(\omega)) d \mathbb{P}(\omega)
$$

for all $\ell \in \mathcal{X}^{*}$, where $\mathcal{X}^{*}$ stands for the dual of $\mathcal{X}$. Following the standard notation, we write

$$
\mathbb{E}[X]=\int_{\Omega} X(\omega) d \mathbb{P}(\omega) .
$$

We call $\mathbb{E}[X]$ the Bochner integral of $X$ with respect to $\mathbb{P}$. More details about the existence and some properties of this integral can be found in [17], [19].

Vol. 37, $\mathrm{N}^{\circ}$ 1, 2019] 
For $\mathcal{X}$-valued random variables, we define the convergence in probability similarly to the real-valued case. If $\left\{X_{j}\right\}$ is a sequence of $\mathcal{X}$-valued random variable, we say that $X_{j}$ converges to a $\mathcal{X}$-valued random variable $X$ in probability if for all $\varepsilon>0$, we have

$$
\lim _{j \rightarrow \infty} \mathbb{P}\left(\left\{\omega \in \Omega:\left\|X_{j}(\omega)-X(\omega)\right\|_{\mathcal{X}} \geq \varepsilon\right\}\right)=0 .
$$

When $X$ is a real-valued random variable, we use the usual notation and denote the expected value of $X$ and its variance by

$$
\mathbb{E}[X]:=\int_{\Omega} X(\omega) d \mathbb{P}(\omega) \text { and } \operatorname{Var} X:=\mathbb{E}\left[(\mathbb{E}[X]-X)^{2}\right],
$$

respectively. For both senses of expectation presented above, we also use the notation

$$
\mathbb{E}_{A}[X]=\int_{A} X(\omega) d \mathbb{P}(\omega)
$$

when $A \subset \Omega$ is measurable and the right-hand-side of (10) makes sense.

Let $X$ and $Y$ be two $E$-valued random variable in the same probability space. We say that they are identically distributed if for all $A \in \mathcal{E}$ we have

$$
\mathbb{P}\left(X^{-1}(A)\right)=\mathbb{P}\left(Y^{-1}(A)\right) .
$$

Now we introduce the notion of independence. Given a finite set of random variables $X_{1}, \ldots X_{j}$, they are said to be independent if for all $A_{i} \in \mathcal{E}, 1 \leq i \leq j$, we have

$$
\mathbb{P}\left(\cap_{i=1}^{j} X_{i} \in A_{i}\right)=\prod_{i=1}^{j} \mathbb{P}\left(X_{i} \in A_{i}\right) .
$$

Finally, a sequence of random variables $\left\{X_{1}, X_{2}, \ldots\right\}$ is independent if all finite collection of this sequence form a set of independent random variables.

\section{Main results and proofs}

Let $G$ be the Green function of the Laplacian operator $-\Delta$ in the bounded domain $U \subset \mathbb{R}^{n}$ with $n \geq 3$. It is known that (see [9]), for all $x, y \in U$, there holds

$$
0 \leq G(x, y) \leq \frac{1}{n \alpha_{n}(n-2)} \frac{1}{|x-y|^{n-2}},
$$

where $\alpha_{n}$ stands for the volume of the unit ball in $\mathbb{R}^{n}$. Hence, if we denote by $d_{U}$ the diameter of $U$, namely

$$
d_{U}:=\sup _{x_{1}, x_{2} \in U}\left|x_{1}-x_{2}\right|,
$$

and

$$
B_{d_{U}}(x)=\left\{y \in \mathbb{R}^{n} ;|y-x|<d_{U}\right\},
$$

a straightforward calculation provides

[Revista Integración, temas de matemáticas 


$$
\begin{aligned}
\int_{U} G(x, y) d y & \leq \frac{1}{n \alpha_{n}(n-2)} \int_{B_{d_{U}}(x)} \frac{1}{|x-y|^{n-2}} d y \\
& =\frac{1}{n \alpha_{n}(n-2)} \frac{n \alpha_{n} d_{U}^{2}}{2}=\frac{d_{U}^{2}}{2(n-2)},
\end{aligned}
$$

for all $x \in U$. From now on, we write only $l_{0}=l_{0}(n, U)$ to denote the quantity

$$
l_{0}:=\frac{d_{U}^{2}}{2(n-2)} .
$$

Inequality (11) implies that the map $H: L^{\infty}(U) \rightarrow L^{\infty}(U)$ given by

$$
H(\varphi)(x):=\int_{U} G(x, y) \varphi(y) d y, \quad x \in U,
$$

is well-defined. More specifically, for any $\varphi \in L^{\infty}(U)$, we have that

$$
|H(\varphi)(x)| \leq \int_{U} G(x, y)|\varphi(y)| d y \leq\|\varphi\|_{\infty} \int_{U} G(x, y) d y
$$

and then

$$
\|H(\varphi)\|_{\infty} \leq l_{0}\|\varphi\|_{\infty}
$$

Standard calculations show that the problem (3) is formally equivalent to the integral equation

$$
u(x)=H(g)-H\left(V_{\omega} u\right)+H\left(b u|u|^{p-1}\right) .
$$

A solution of (14) is called an integral solution of (3).

In what follows, we give estimates for the terms of (14) in order to apply a fixed point argument. We first set $\mathcal{X}:=L^{\infty}(U)$ and define, for any fixed $\omega \in \Omega$, the linear function $T: \mathcal{X} \rightarrow \mathcal{X}$ given by

$$
T(u):=-H\left(V_{\omega} u\right), \quad \forall u \in \mathcal{X} .
$$

It follows from (13) and (8) that, for any $u \in \mathcal{X}$, there holds

$$
\|T(u)\|_{\infty} \leq l_{0}\left\|V_{\omega} u\right\|_{\infty} \leq l_{0}\|f\|_{\infty}\left|\mu_{\omega}\right|\|u\|_{\infty}
$$

and so

$$
\|T\|_{\mathcal{X} \rightarrow \mathcal{X}} \leq l_{0}\|f\|_{\infty}\left|\mu_{\omega}\right|
$$

For the nonlinear term in (14), we define $B: \mathcal{X} \rightarrow \mathcal{X}$ by

$$
B(u):=H\left(b|u|^{p-1} u\right), \quad \forall u \in \mathcal{X} .
$$

For $a_{1}, a_{2} \in \mathbb{R}$, there holds

$$
\left.\left|a_{1}\right| a_{1}\right|^{p-1}-a_{2}\left|a_{2}\right|^{p-1}|\leq p| a_{1}-a_{2} \mid\left(\left|a_{1}\right|^{p-1}+\left|a_{2}\right|^{p-1}\right),
$$

and then it follows that

$$
\left\|b(\cdot)\left(u|u|^{p-1}-\tilde{u}|\tilde{u}|^{p-1}\right)\right\|_{\infty} \leq\|b\|_{\infty}\|u-\tilde{u}\|_{\infty}\left(\|u\|_{\infty}^{p-1}+\|\tilde{u}\|_{\infty}^{p-1}\right) .
$$

Vol. 37, $\mathrm{N}^{\circ}$ 1, 2019] 
This inequality and the same argument used in (15) imply that

$$
\|B(u)-B(\tilde{u})\|_{\infty} \leq l_{0} p\|b\|_{\infty}\|u-\tilde{u}\|_{\infty}\left(\|u\|_{\infty}^{p-1}+\|\tilde{u}\|_{\infty}^{p-1}\right),
$$

for any $u, \tilde{u} \in L^{\infty}(U)$.

All together, the above estimates enable us to solve the random equation (3) as follows.

Proposition 3.1. Given $f, b, g \in L^{\infty}(U)$ and $\omega \in \Omega$, we consider the potential $V_{\omega}$ induced by the random measure $\mu_{\omega}:=X(\omega)$. Let $l_{0}$ be the quantity introduced in (12) and set

$$
\tau_{\omega}:=l_{0}\|f\|_{\infty}\left|\mu_{\omega}\right| \quad \text { and } K:=l_{0} p\|b\|_{\infty} .
$$

If $\varepsilon>0$ and $\omega \in \Omega$ are such that

$$
0 \leq \tau_{\omega}<1, \quad \frac{2^{p} K \varepsilon^{p-1}}{\left(1-\tau_{\omega}\right)^{p-1}}+\tau_{\omega}<1
$$

and $\|g\|_{\infty} \leq \varepsilon / l_{0}$, then the equation (3) has a unique integral solution $u_{\omega}$ (i.e. it satisfies (14)) such that

$$
u_{\omega}=u(\cdot, \omega) \in L^{\infty}(U) \text { and }\left\|u_{\omega}\right\|_{\infty} \leq \frac{2 \varepsilon}{1-\tau_{\omega}} .
$$

Proof. For each $\varepsilon>0$ and $\omega \in \Omega$ satisfying (18), we consider the closed ball

$$
\mathcal{B}_{\varepsilon}=\left\{u \in L^{\infty}(U) ;\|u\|_{\infty} \leq \frac{2 \varepsilon}{\left(1-\tau_{\omega}\right)}\right\}
$$

endowed with the metric $d(u, v):=\|u-v\|_{\infty}$. We are going to show that the map

$$
\Phi(u):=H(g)-H\left(V_{\omega} u\right)+H\left(b u|u|^{p-1}\right)=H(g)+T(u)+B(u)
$$

is a contraction on the complete metric space $\left(\mathcal{B}_{\varepsilon}, d\right)$. Using the estimates $(13),(15)$, and (16) with $\tilde{u}=0$, we obtain

$$
\begin{aligned}
\|\Phi(u)\|_{\infty} & \leq\|H(g)\|_{\infty}+\|T(u)\|_{\infty}+\|B(u)\|_{\infty} \\
& \leq l_{0}\|g\|_{\infty}+\tau_{\omega}\|u\|_{\infty}+K\|u\|_{\infty}^{p} \\
& \leq \varepsilon+\tau_{\omega} \frac{2 \varepsilon}{1-\tau_{\omega}}+\frac{2^{p} K \varepsilon^{p}}{\left(1-\tau_{\omega}\right)^{p}} \\
& =\left(1+\tau_{\omega}+\frac{2^{p} K \varepsilon^{p-1}}{\left(1-\tau_{\omega}\right)^{p-1}}\right) \frac{\varepsilon}{1-\tau_{\omega}}
\end{aligned}
$$

for all $u \in \mathcal{B}_{\varepsilon}$. Hence, it follows from (18) that

$$
\|\Phi(u)\|_{\infty} \leq \frac{2 \varepsilon}{1-\tau_{\omega}} .
$$

This shows that $\Phi$ maps $\mathcal{B}_{\varepsilon}$ into $\mathcal{B}_{\varepsilon}$.

[Revista Integración, temas de matemáticas 
For all $u, \widetilde{u} \in \mathcal{B}_{\varepsilon}$, it follows from (15) and (16) that

$$
\begin{aligned}
\|\Phi(u)-\Phi(\widetilde{u})\|_{\infty} & =\|T(u-\widetilde{u})\|_{\infty}+\|B(u)-B(\widetilde{u})\|_{\infty} \\
& \leq \tau_{\omega}\|u-\widetilde{u}\|_{\infty}+K\|u-\widetilde{u}\|_{\infty}\left(\|u\|_{\infty}^{p-1}+\|\widetilde{u}\|_{\infty}^{p-1}\right) \\
& \leq\left(\tau_{\omega}+\frac{2^{p} K \varepsilon^{p-1}}{\left(1-\tau_{\omega}\right)^{p-1}}\right)\|u-\widetilde{u}\|_{\infty} .
\end{aligned}
$$

In view of (18), the above estimate implies that $\Phi$ is a contraction in $\mathcal{B}_{\varepsilon}$. Now, the Banach fixed point theorem assures that there is a unique solution $u$ for the integral equation (14) such that $\|u\|_{\infty} \leq(2 \varepsilon) /\left(1-\tau_{\omega}\right)$.

The next results are related to the randomness introduced by the random potential $V$ and existence of solutions for the problem (3). Roughly speaking, we first obtain the probability of (3) having a solution via the method discussed above. In the sequel we discuss a law of large numbers for a sequence of random potentials.

Theorem 3.2. Let $\nu$ be the probability measure induced on $\mathbb{R}$ by the random variable $\omega \mapsto\left|\mu_{\omega}\right|$. Let $g \in L^{\infty}(U)$ be such that $\|g\|_{\infty}<\frac{1}{l_{0}}\left(\frac{1}{2^{p} K}\right)^{\frac{1}{p-1}}$, where $K=l_{0} p\|b\|_{\infty}$. Choose $0<c_{0}<1$ in such a way that $\|g\|_{\infty} \leq \frac{\varepsilon_{0}}{l_{0}}$ with

$$
\varepsilon_{0}:=\left(\frac{\left(1-c_{0}\right)^{p}}{2^{p} K}\right)^{\frac{1}{p-1}} .
$$

Let $\mathcal{A}$ be the set of $\omega \in \Omega$ such that (3) has a solution $u(\cdot, \omega)$ given by Proposition 3.1 with $\varepsilon=\varepsilon_{0}$. The set $\mathcal{A}$ is called the admissible one for the random variable $X(\omega)$ and non-homogeneous term $g$.

(i) The set $\mathcal{A}$ is $\mathcal{F}$-measurable, and the probability of (3) having a solution is

$$
\mathbb{P}(\mathcal{A})=\nu\left(\left[0, \frac{c_{0}}{l_{0}\|f\|_{\infty}}\right)\right) .
$$

(ii) Let $u_{\omega}, \tilde{u}_{\omega}$ be two solutions of (3) corresponding, respectively, to $\mu_{\omega}, g, \mathcal{A}$ and $\tilde{\mu}_{\omega}, \tilde{g}, \widetilde{\mathcal{A}}$ with $\|g\|_{\infty},\|\tilde{g}\|_{\infty} \leq \frac{\varepsilon_{0}}{l_{0}}$. Assume that $\mathcal{A} \cap \widetilde{\mathcal{A}} \neq \varnothing$ and define

$$
\eta_{\omega}:=l_{0}\|f\|_{\infty} \max \left\{\left|\mu_{\omega}\right|,\left|\widetilde{\mu}_{\omega}\right|\right\}, \text { for } \omega \in \mathcal{A} \cap \widetilde{\mathcal{A}} .
$$

We have that

$$
\|u(\cdot, \omega)-\tilde{u}(\cdot, \omega)\|_{\infty} \leq \frac{l_{0}\left(\|g-\tilde{g}\|_{\infty}+\frac{2 \varepsilon_{0}}{1-\eta_{\omega}}\|f\|_{\infty} \mid \mu_{\omega}-\tilde{\mu}_{\omega} \mathbf{I}\right)}{1-\eta_{\omega}-\frac{2^{p} K \varepsilon_{0}^{p-1}}{\left(1-\eta_{\omega}\right)^{p-1}}}
$$

for all $\omega \in \mathcal{A} \cap \widetilde{\mathcal{A}}$.

Vol. 37, $\left.\mathrm{N}^{\circ} 1,2019\right]$ 
(iii) The map $\mathcal{U}: \mathcal{A} \rightarrow L^{\infty}(U)$ given by $\mathcal{U}(\omega):=u(\cdot, \omega)$ is a random variable, and there holds

$$
\|\mathcal{U}(\omega)\|_{\infty} \leq \frac{2 \varepsilon_{0}}{1-\tau_{\omega}}=2 \varepsilon_{0} \sum_{j=0}^{\infty} \tau_{\omega}^{j}
$$

for all $\omega \in \mathcal{A}$.

Proof. We first notice that $\omega \in \mathcal{A}$ if only if $\tau_{\omega}=l_{0}\|f\|_{\infty}\left|\mu_{\omega}\right|$ verifies (18) with $\varepsilon=\varepsilon_{0}$. Then, if $Y(\omega)=|X(\omega)|=\left|\mu_{\omega}\right|$, it follows that $\mathcal{A}=\left\{Y \in\left[0, \frac{c_{0}}{l_{0}\|f\|_{\infty}}\right)\right\}$ is measurable and

$$
\begin{aligned}
\mathbb{P}(\mathcal{A}) & =\mathbb{P}\left(Y \in\left[0, \frac{c_{0}}{l_{0}\|f\|_{\infty}}\right)\right) \\
& =\mathbb{P}_{Y}\left(\left[0, \frac{c_{0}}{l_{0}\|f\|_{\infty}}\right)\right) \\
& =\nu\left(\left[0, \frac{c_{0}}{l_{0}\|f\|_{\infty}}\right)\right) .
\end{aligned}
$$

This establishes (i).

Now we deal with item (ii). Firstly, observe that $\eta_{\omega}=\max \left\{\tau_{\omega}, \widetilde{\tau}_{\omega}\right\}$, where

$$
\tau_{\omega}=l_{0}\|f\|_{\infty}\left|\mu_{\omega}\right| \text { and } \tilde{\tau}_{\omega}=l_{0}\|f\|_{\infty}\left|\tilde{\mu}_{\omega}\right|
$$

Subtracting the integral equations verified by $u_{\omega}$ and $\tilde{u}_{\omega}$, and afterwards computing $\|\cdot\|_{\infty}$, we obtain

$$
\begin{aligned}
\left\|u_{\omega}-\tilde{u}_{\omega}\right\|_{\infty} \leq & \|H(g-\tilde{g})\|_{\infty}+\left\|H\left(V_{\omega}\left(u-\tilde{u}_{\omega}\right)\right)\right\|_{\infty} \\
& +\left\|H\left(\left(V_{\omega}-\widetilde{V}_{\omega}\right) \tilde{u}_{\omega}\right)\right\|_{\infty} \\
& +\left\|H\left(b\left(u_{\omega}\left|u_{\omega}\right|^{p-1}-\tilde{u}_{\omega}\left|\tilde{u}_{\omega}\right|^{p-1}\right)\right)\right\|_{\infty} \\
\leq & l_{0}\|g-\tilde{g}\|_{\infty}+l_{0}\|f\|_{\infty} \mid \mu_{\omega}\left\|u_{\omega}-\tilde{u}_{\omega}\right\|_{\infty} \\
& +l_{0}\|f\|_{\infty} \mid \mu_{\omega}-\tilde{\mu}_{\omega}\left\|\tilde{u}_{\omega}\right\|_{\infty} \\
& +l_{0} p\|b\|_{\infty}\left\|u_{\omega}-\tilde{u}_{\omega}\right\|_{\infty}\left(\left\|u_{\omega}\right\|_{\infty}^{p-1}+\left\|\tilde{u}_{\omega}\right\|_{\infty}^{p-1}\right) .
\end{aligned}
$$

It follows from (19) that

$$
\left\|u_{\omega}\right\|_{\infty} \leq \frac{2 \varepsilon_{0}}{1-\tau_{\omega}} \leq \frac{2 \varepsilon_{0}}{1-\eta_{\omega}} \text { and }\|\tilde{u}\|_{\infty} \leq \frac{2 \varepsilon_{0}}{1-\widetilde{\tau}_{\omega}} \leq \frac{2 \varepsilon_{0}}{1-\eta_{\omega}} .
$$


The two above expressions give us

$$
\begin{aligned}
\left\|u_{\omega}-\tilde{u}_{\omega}\right\|_{\infty} \leq & l_{0}\|g-\tilde{g}\|_{\infty}+l_{0}\|f\|_{\infty}\left|\mu_{\omega}\right|\left\|u_{\omega}-\tilde{u}_{\omega}\right\|_{\infty} \\
& +l_{0} \frac{2 \varepsilon_{0}}{1-\eta_{\omega}}\|f\|_{\infty}\left|\mu_{\omega}-\tilde{\mu}_{\omega}\right|+\frac{2^{p} K \varepsilon_{0}^{p-1}}{\left(1-\eta_{\omega}\right)^{p-1}}\left\|u_{\omega}-\tilde{u}_{\omega}\right\|_{\infty} \\
= & l_{0}\|g-\tilde{g}\|_{\infty}+l_{0} \frac{2 \varepsilon_{0}}{1-\eta_{\omega}}\|f\|_{\infty}\left|\mu_{\omega}-\tilde{\mu}_{\omega}\right| \\
& +\left[\eta_{\omega}+\frac{2^{p} K \varepsilon_{0}^{p-1}}{\left(1-\eta_{\omega}\right)^{p-1}}\right]\left\|u_{\omega}-\tilde{u}_{\omega}\right\|_{\infty},
\end{aligned}
$$

which yields (21).

Taking $\mu_{\omega}, \tilde{\mu}_{\omega}$ independent of $\omega$, i.e. $\mu_{\omega}=\mu$ and $\tilde{\mu}_{\omega}=\tilde{\mu}$, for all $\omega \in \Omega$, we can see from (17) and (21) that the data-map solution $\mathcal{L}(\mu, g)=u$ is continuous from

$$
\left\{(\mu, g) \in \mathcal{M}(U) \times L^{\infty}(U) ;|\mu|<\frac{c_{0}}{l_{0}\|f\|_{\infty}},\|g\|_{\infty} \leq \frac{\varepsilon_{0}}{l_{0}}\right\} \text { to } L^{\infty}(U),
$$

where $u$ is the deterministic solution of (3) corresponding to the data $(\mu, g)$. From this, and because $\left.X\right|_{\mathcal{A}}$ given by $X(\omega)=\mu_{\omega}$ is measurable, it follows that the composition $\mathcal{U}(\omega)=\mathcal{L}\left(\mu_{\omega}, g\right)=\mathcal{L}(X(\omega), g)$ from $\mathcal{A}$ to $L^{\infty}(U)$ is measurable.

In view of the series $\frac{1}{1-z}=\sum_{j=0}^{\infty} z^{j}$ for $|z|<1$, we finish by observing that (22) follows from (19) with $\varepsilon=\varepsilon_{0}$ and $\omega \in \mathcal{A}$.

Remark 3.3. Here we give examples of random potentials for which there exists a solution almost surely in $\Omega$. The first occurs if we suppose that the measure $\nu$ has compact support contained in the interval $[0, a]$, with $a<\frac{c_{0}}{l_{0}\|f\|_{\infty}}$. In this case it follows from item $(i)$ of Theorem 3.2 that $\mathbb{P}(\mathcal{A})=1$, i.e., the solution exists almost surely in $\Omega$. Second, take a sequence $\left\{\mu_{j}\right\}_{j \in \mathbb{N}}$ in $\mathcal{M}(U)$, and let $\left\{a_{j}(\omega)\right\}_{j \in \mathbb{N}}$ be a sequence of random variables from $\Omega$ to $\mathbb{R}$. Consider the random variable $\mu_{\omega}$ defined by

$$
\mu_{\omega}=\sum_{j=1}^{\infty} a_{j}(\omega) \mu_{j} .
$$

For some $q>1$, suppose that

$$
\left|a_{j}(\omega)\right|<\frac{\left(\sum_{k=1}^{\infty} \frac{1}{k^{q}}\right)^{-1}}{l_{0}\left|\mu_{j}\right|\|f\|_{\infty}} \frac{c_{0}}{j^{q}} \text { a.s. in } \Omega,
$$

for all $j \in \mathbb{N}$. Then,

$$
\left|\mu_{\omega}\right| \leq \sum_{j=1}^{\infty}\left|a_{j}(\omega) \| \mu_{j}\right|<\frac{c_{0}}{l_{0}\|f\|_{\infty}} \text { a.s. in } \Omega
$$

and Theorem 3.2 assures that there is an integral solution for (3) a.s. in $\Omega$.

Vol. 37, $\left.\mathrm{N}^{\circ} 1,2019\right]$ 
In the sequel we show how the Borel-Cantelli's Lemma can be used to give a sufficient condition for the existence of solution a.s. in $\Omega$.

Corollary 3.4. Let $c_{0}$ and $g$ be as in Theorem 3.2. Let $\left\{\mu_{j}\right\}_{j \in \mathbb{N}}$ be a sequence in $\mathcal{M}(U)$ and let $\left\{a_{j}(\omega)\right\}_{j \in \mathbb{N}}$ be a sequence of random variables from $\Omega$ to $\mathbb{R}$. Assume that the series

$$
\mu_{\omega}=\sum_{j=1}^{\infty} a_{j}(\omega) \mu_{j}
$$

is convergent in $\mathcal{M}(U)$.

For each $k \in \mathbb{N}$, define

$$
S_{k}(\omega)=\sum_{j=1}^{k} a_{j}(\omega) \mu_{j}
$$

and $L_{k}=\left\{\omega \in \Omega:\left|S_{k}\right| \geq \tilde{c}\right\}$, with $0<\tilde{c}<c_{0} /\left(l_{0}\|f\|_{\infty}\right)$. If

$$
\sum_{k=1}^{\infty} \mathbb{P}\left(L_{k}\right)<\infty
$$

then there is an integral solution for (3) almost surely in $\Omega$.

Proof. By Borel-Cantelli's Lemma we get that $\mathbb{P}\left(\lim \sup L_{k}\right)=0$, that is,

$$
\mathbb{P}\left(\cup_{j=1}^{\infty} \cap_{k=j}^{\infty}\left\{\left|S_{k}\right|<\tilde{c}\right\}\right)=1 .
$$

It follows that, for almost sure $\omega \in \Omega$, there is $j_{0}=j_{0}(\omega)$ such that for all $j>j_{0}$, we have

$$
\left|S_{j}(\omega)\right|<\tilde{c}
$$

Therefore, by taking the limit as $j \rightarrow \infty$, we obtain

$$
\left|\mu_{\omega}\right|=\lim _{j \rightarrow \infty}\left|S_{j}(\omega)\right| \leq \tilde{c}<\frac{c_{0}}{l_{0}\|f\|_{\infty}} \quad \text { a.s. in } \Omega .
$$

This inequality and Theorem 3.2 imply that there is an integral solution $u(x, \omega)$ for $(3)$ almost surely in $\Omega$.

A straightforward calculation shows that in general $\mathbb{E}_{\Omega}(u(x, \omega))$ does not satisfy the equation (3), even if we replace the random potential by its mean. However, we are able to obtain some information on the average and moments of the random solution $u_{\omega}$. Let us mention that, when dealing with the random variable $\omega \mapsto u_{\omega}$, the expectation has to be understood in the Bochner sense (see Section 2). Note also that in fact a solution $u_{\omega} \in L^{\infty}(U)$ of (14) belongs to the separable subspace $C(\bar{U})$.

Theorem 3.5. Assume the hypotheses of Theorem 3.2 and denote by $u_{\omega}(x)=u(x, \omega) \in$ $L^{\infty}(U)$ the solution of (3). Let $m \in \mathbb{N}$ be fixed and suppose that

$$
\sum_{j=1}^{\infty} \frac{(m+j-1) !}{(m-1) ! j !}\left(l_{0}\|f\|_{\infty}\right)^{j} \mathbb{E}_{\mathcal{A}}\left[\mid \mathbf{|} \mu_{\omega} \mathbf{|}^{j}\right]<+\infty .
$$

[Revista Integración, temas de matemáticas 
Then $\mathbb{E}_{\mathcal{A}}\left[|u|^{m}(x, \omega)\right] \in L^{\infty}(U)$ and

$$
\mathbb{E}_{\mathcal{A}}\left[\left\||u|^{m}(\cdot, \omega)\right\|_{L^{\infty}(U)}\right]<\infty .
$$

In the case $m=1$, we have $\mathbb{E}_{\mathcal{A}}[u(x, \omega)] \in L^{\infty}(U)$ and

$$
\mathbb{E}_{\mathcal{A}}\left[\|u(\cdot, \omega)\|_{L^{\infty}(U)}\right] \leq\left(2 \varepsilon_{0}\right)\left(1+\sum_{j=1}^{\infty}\left(l_{0}\|f\|_{\infty}\right)^{j} \mathbb{E}_{\mathcal{A}}\left[\left|\mu_{\omega}\right|^{j}\right]\right) .
$$

Proof. It follows from (22) that

$$
\left\||u|^{m}(\cdot, \omega)\right\|_{L^{\infty}(U)} \leq\|u(\cdot, \omega)\|_{L^{\infty}(U)}^{m} \leq \frac{\left(2 \varepsilon_{0}\right)^{m}}{\left(1-\tau_{\omega}\right)^{m}} .
$$

Computing $\mathbb{E}_{\mathcal{A}}$ in (27), we obtain

$$
\begin{aligned}
\left\|\mathbb{E}_{\mathcal{A}}\left[|u|^{m}(x, \omega)\right]\right\|_{L^{\infty}(U)} & \leq \mathbb{E}_{\mathcal{A}}\left[\left\||u|^{m}(x, \omega)\right\|_{L^{\infty}(U)}\right] \\
& \leq\left(2 \varepsilon_{0}\right)^{m} \mathbb{E}_{\mathcal{A}}\left[\left(1+\sum_{j=1}^{\infty} \frac{(m+j-1) !}{(m-1) ! j !} \tau_{\omega}^{j}\right)\right] .
\end{aligned}
$$

By using the linearity of the expectation and recalling that $\tau_{\omega}=l_{0}\|f\|_{\infty}\left|\mu_{\omega}\right|$, we get the following upper bound for the right hand side of (28):

$$
\left(2 \varepsilon_{0}\right)^{m}+\left(2 \varepsilon_{0}\right)^{m} \sum_{j=1}^{\infty} \frac{(m+j-1) !}{(m-1) ! j !}\left(l_{0}\|f\|_{\infty}\right)^{j} \mathbb{E}_{\mathcal{A}}\left[\left|\mu_{\omega}\right|^{j}\right]
$$

this bound is finite due to (24). From (25) with $m=1$ and the estimate

$$
\left\|\mathbb{E}_{\mathcal{A}}[u(x, \omega)]\right\|_{L^{\infty}(U)} \leq \mathbb{E}_{\mathcal{A}}\left[\||u|(x, \omega)\|_{L^{\infty}(U)}\right],
$$

we obtain that $\mathbb{E}_{\mathcal{A}}[u(\cdot, \omega)] \in L^{\infty}(U)$. The estimate (26) follows by taking $m=1$ in (28)-(29) and afterwards using (30).

In the sequel we show a weak law of large numbers for the random $L^{\infty}(U)$-solutions obtained in Section 2.

Theorem 3.6. Let $\left\{X_{j}\right\}_{j \in \mathbb{N}}$ be an independent sequence of random variables $X_{j}: \Omega \rightarrow$ $\mathcal{M}(U)$. Assume that the admissible set $\mathcal{A}_{j}=\Omega$ for all $j$, and let $u_{j}(\cdot, \omega) \in L^{\infty}(U)$ be the solution given by Theorem 3.2 with respect to $X_{j}(\omega)=\mu_{\omega, j}$ and $g$. If $X_{j} \rightarrow X$ a.s. and

$$
L=\sup _{j \in \mathbb{N}}\left(\operatorname{ess} \sup _{\omega \in \Omega}\left|\mu_{\omega, j}\right|\right)<\frac{c_{0}}{l_{0}\|f\|_{\infty}}
$$

then

$$
\sum_{j=1}^{k} \frac{u_{j}(x, \omega)-\mathbb{E}_{\Omega}\left[u_{j}(x, \omega)\right]}{k} \rightarrow 0
$$

Vol. 37, $\left.\mathrm{N}^{\circ} 1,2019\right]$ 
and

$$
\sum_{j=1}^{k} \frac{\left\|u_{j}(\cdot, \omega)\right\|_{\infty}-\mathbb{E}_{\Omega}\left[\left\|u_{j}(\cdot, \omega)\right\|_{\infty}\right]}{k} \rightarrow 0
$$

as $k \rightarrow \infty$, where the convergences in (32) and (33) are in the sense of probability (see $(9))$.

Proof. Notice that $X_{j} \rightarrow X$ a.s. is equivalent to $\mu_{\omega, j} \rightarrow \mu_{\omega}=X(\omega)$ in $\mathcal{M}(U)$ almost surely. From this and the continuity of data-solution map $\mathcal{L}(\cdot, \cdot)$ (see $(23)$ ), it follows that

$$
\left\|u_{j}(\cdot, \omega)-u(\cdot, \omega)\right\|_{\infty}=\left\|\mathcal{L}\left(\mu_{\omega, j}, g\right)-\mathcal{L}(\mu, g)\right\|_{\infty} \rightarrow 0,
$$

as $j \rightarrow \infty$. Recalling (22) and afterwards using (31), we obtain

$$
\begin{aligned}
\left\|u_{j}(\cdot, \omega)\right\|_{\infty} & \leq \frac{2 \varepsilon_{0}}{1-l_{0}\|f\|_{\infty}\left(\operatorname{ess} \sup _{\omega \in \Omega} \mid \mu_{\omega, j} \mathbf{l}\right)} \\
& \leq \frac{2 \varepsilon_{0}}{1-L}=Q_{0}, \text { a.s. in } \Omega .
\end{aligned}
$$

Next, for a fixed $g$ such that $\|g\|_{\infty} \leq \frac{\varepsilon_{0}}{l_{0}}$, consider

$$
S_{g}(\mu)=\mathcal{L}(\mu, g)
$$

defined from $D$ to $L^{\infty}(U)$, where $D=\left\{\mu \in \mathcal{M}(U):|\mu|<\frac{c_{0}}{l_{0}\|f\|_{\infty}}\right\}$. Since $X_{j}$ 's are independent, it follows that $\left\{Y_{j}\right\}_{j \in \mathbb{N}}$ defined by

$$
Y_{j}=\left\|u_{j}(\cdot, \omega)\right\|_{\infty}=\left\|S_{g} \circ X_{j}(\omega)\right\|_{\infty}
$$

are also independent. So, from Chebyshev's inequality, the independence of $\left\{Y_{j}\right\}_{j \in \mathbb{N}}$, and (34), we have that

$$
\begin{aligned}
\mathbb{P}\left(\left|k^{-1} \sum_{j=1}^{k}\left(\left\|u_{j}(\cdot, \omega)\right\|_{\infty}-\mathbb{E}_{\Omega}\left[\left\|u_{j}(\cdot, \omega)\right\|_{\infty}\right]\right)\right| \geq \delta\right) \\
\leq \frac{1}{(k \delta)^{2}} \mathbb{E}_{\Omega}\left[\left|\sum_{j=1}^{k}\left(\left\|u_{j}(\cdot, \omega)\right\|_{\infty}-\mathbb{E}_{\Omega}\left[\left\|u_{j}(\cdot, \omega)\right\|_{\infty}\right]\right)\right|^{2}\right] \\
=\frac{1}{(k \delta)^{2}} \sum_{j=1}^{k} \mathbb{E}_{\Omega}\left[\left|\left(\left\|u_{j}(\cdot, \omega)\right\|_{\infty}-\mathbb{E}_{\Omega}\left[\left\|u_{j}(\cdot, \omega)\right\|_{\infty}\right]\right)\right|^{2}\right] \\
\leq \frac{1}{(k \delta)^{2}} \sum_{j=1}^{k} \mathbb{E}_{\Omega}\left[\left|2 Q_{0}\right|^{2}\right] \\
\leq \frac{1}{(k \delta)^{2}} \sum_{j=1}^{k}\left|2 Q_{0}\right|^{2} \mathbb{E}_{\Omega}[1] \\
\leq \frac{4 Q_{0}^{2}}{\delta^{2}} \frac{1}{k}
\end{aligned}
$$

[Revista Integración, temas de matemáticas 
Letting $k \rightarrow+\infty$ in the above expression, we get (33). The convergence (32) can be proved similarly to $(33)$.

Acknowledgments. L.C.F. Ferreira was supported by FAPESP-SP and CNPq, Brazil. M. Furtado and L. Cioletti were supported by CNPq, Brazil.

\section{References}

[1] Ambrosetti A., Badiale M. and Cingolani S., "Semiclassical states of nonlinear Schrödinger equations", Arch. Ration. Mech. Anal. 140 (1997), No. 3, 285-300.

[2] Ambrosetti A., Malchiodi A. and Secchi S., "Multiplicity results for some nonlinear Schrödinger equations with potentials", Arch. Ration. Mech. Anal. 159 (2001), No.3, 253-271.

[3] Bal G., Komorowski T. and Ryzhik L., "Asymptotic of the Solutions of the Random Schrödinger Equation", Arch. Ration. Mech. Anal. 200 (2011), No.2, 613-664.

[4] Beck L. and Flandoli F., "A regularity theorem for quasilinear parabolic systems under random perturbations", J. Evol. Equ. 13 (2013), No. 4, 829-874.

[5] Bourgain J., "Nonlinear Schrödinger Equation with a Random Potential", Illinois J. Math. 50 (2006), No. 1-4, 183-188.

[6] Conlon J.G. and Naddaf A., "Green's Functions for Elliptic and Parabolic Equations with Random Coefficients", New York J. Math. 6 (2000), 153-225.

[7] Dawson D.A. and Kouritzin M., "Invariance Principles for Parabolic Equations with Random Coefficients", J. Funct. Anal. 149 (1997), No. 2, 377-414.

[8] Del Pino M. and Felmer P., "Local Mountain Pass for semilinear elliptic problems in unbounded domains", Calc. Var. Partial Differential Equations 4 (1996), No. 2, $121-137$.

[9] Evans L.C., Partial differential equations, Graduate Studies in Mathematics 19, Amer. Math. Soc., Providence, RI, 1998.

[10] Fannjiang A., "Self-Averaging Scaling Limits for Random Parabolic Waves", Arch. Ratio. Mech. Anal. 175 (2008), No. 3, 343-387.

[11] Ferreira L.C.F. and Castañeda-Centurión N.F., "A Fourier analysis approach to elliptic equations with critical potentials and nonlinear derivative terms", Milan J. Math. 85 (2017), No. 2, 187-213.

[12] Ferreira L.C.F. and Mesquita C.A.A.S., "Existence and symmetries for elliptic equations with multipolar potentials and polyharmonic operators", Indiana Univ. Math. J. 62 (2013), No. 6, 1955-1982.

[13] Ferreira L.C.F. and Montenegro M., "A Fourier approach for nonlinear equations with singular data", Israel J. Math. 193 (2013), No. 1, 83-107.

Vol. 37, $\mathrm{N}^{\circ}$ 1, 2019] 
[14] Ferreira L.C.F. and Montenegro M., "Existence and asymptotic behavior for elliptic equations with singular anisotropic potentials", J. Differential Equations 250 (2011), No.4, 2045-2063.

[15] Ferreira L.C.F., Medeiros E.S. and Montenegro M., "A class of elliptic equations in anisotropic spaces", Ann. Mat. Pura Appl. 192 (2013), No. 4, 539-552.

[16] Flandoli F., "Random perturbation of PDEs and fluid dynamic models", in Saint Flour Summer School Lectures 2010, Lecture Notes in Math., Springer (2011), Heidelberg, 1-176.

[17] Hille E. and Phillips R.S., Functional Analysis and Semigroups, Amer. Math Soc. Colloquium Publ. 31, Amer. Math. Soc., Providence, Rhode, 1957.

[18] Kirsch W., "An invitation to random Schrödinger operators", in Random Schrödinger operators, Vol. 25 of Panor. Synthèses, Soc. Math. France, Paris, (2008), 1-119.

[19] Parthasarathy K.R., Probability Measures on Metric Spaces, Academic Press, Providence, 1967.

[20] Rabinowitz P.H., "On a class of nonlinear Schrödinger equations", Z. Angew Math. Phys. 43 (1992), No. 2, 270-291.

[21] Safronov O., "Absolutely continuous spectrum of one random elliptic operator", $J$. Funct. Anal. 255 (2008), No. 3, 755-767. 